\title{
Nano-Computed Tomography: Technique and Applications
}

\section{Nanocomputertomografie: Technik und Applikationen}

Authors

Affiliations
M. Kampschulte ${ }^{1}$, A. C. Langheinirch ${ }^{2}$, J. Sender ${ }^{1}$, H. D. Litzlbauer ${ }^{1}$, U. Althöhn ${ }^{1}$, J. D. Schwab ${ }^{1}$, E. Alejandre-Lafont ${ }^{1}$, G. Martels ${ }^{1}$, G. A. Krombach ${ }^{1}$

Department of Diagnostic and Interventional Radiology, University Hospital Gießen, Germany

2 Department of Diagnostic and Interventional Radiology, BG Trauma Hospital Frankfurt/Main, Germany

\section{Key words \\ nano-CT \\ vasculature \\ bone \\ 3D imaging}

received 4.11.2014

accepted $\quad 5.8 .2015$

\section{Bibliography}

DOI http://dx.doi.org/

10.1055/s-0041-106541

Published online: 2016

Fortschr Röntgenstr 2016; 188:

146-154 @ Georg Thieme

Verlag KG Stuttgart · New York · ISSN 1438-9029

\section{Correspondence \\ Dr. Marian Kampschulte}

Radiologie, Uniklinikum Gießen und Marburg, Standort Gießen Klinikstraße 33

39592 Gießen

Germany

Tel.: ++ 49/641/98541815

Fax: ++49/641/98541809

marian.kampschulte@radiol.

med.uni-giessen.de

\section{Abstract \\ $\nabla$}

Nano-computed tomography (nano-CT) is an emerging, high-resolution cross-sectional imaging technique and represents a technical advancement of the established micro-CT technology. Based on the application of a transmission target X-ray tube, the focal spot size can be decreased down to diameters less than 400 nanometers (nm). Together with specific detectors and examination protocols, a superior spatial resolution up to $400 \mathrm{~nm}$ (10\% MTF) can be achieved, thereby exceeding the resolution capacity of typical micro-CT systems. The technical concept of nano-CT imaging as well as the basics of specimen preparation are demonstrated exemplarily. Characteristics of atherosclerotic plaques (intraplaque hemorrhage and calcifications) in a murine model of atherosclerosis

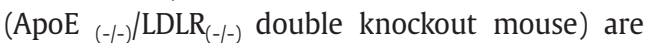
demonstrated in the context of superior spatial resolution in comparison to micro-CT. Furthermore, this article presents the application of nano-CT for imaging cerebral microcirculation (murine), lung structures (porcine), and trabecular microstructure (ovine) in contrast to micro-CT imaging. This review shows the potential of nano-CT as a radiological method in biomedical basic research and discusses the application of experimental, high resolution CT techniques in consideration of other high resolution cross-sectional imaging techniques.

Key Points:

- Nano-computed tomography is a high resolution CT-technology for 3D imaging at sub-micrometer resolution.

- The technical concept bases on a further development of the established ex-vivomicro-CT technology.

- By improvement of the spatial resolution, structures at a cellular level become visible (e.g. osteocyte lacunae).

\section{Citation Format:}

- Kampschulte M, Langheinirch AC, Sender J et al. Nano-Computed Tomography: Technique and Applications. Fortschr Röntgenstr 2016; 188: 146-154

\section{Zusammenfassung}

$\nabla$

Die Nanocomputertomografie (Nano-CT) repräsentiert eine noch junge, hochauflösende Schnittbildtechnologie und stellt eine technische Weiterentwicklung der seit Längerem etablierten Mikrocomputertomografie (Mikro-CT) dar. Durch Einsatz einer Transmissionsröhre, deren Röntgenfokusgröße unterhalb von 400 Nanometer $(\mathrm{nm})$ liegt sowie geeigneter Detektoren und Untersuchungsprotokolle, übertrifft die Nano-CT das räumliche Auflösungsvermögen der klassischen Mikro-CT und ermöglicht eine Ortsauflösung von bis zu $400 \mathrm{~nm}$ (10\% MTF). Exemplarisch werden technisches Konzept der Nano-CT Bildgebung (Strahlen- und Bildentstehung) sowie Grundlagen der Probenmontage vorgestellt. Am Beispiel eines Atherosklerose-Modells, der $\mathrm{ApoE}_{\left.(-)_{-}\right)} / \mathrm{LDLR}_{\left.(-)_{-}\right)}$-Doppel-Knockout-Maus, werden Merkmale atherosklerotischer Plaque, d.h. kalzifizierte sowie hämorrhagische Veränderungen im Kontext der hohen Ortsauflösung und im Vergleich zur Mikro-CT demonstriert. Des Weiteren wird die Anwendung der Nano-CT zur Visualisierung der Mikrozirkulation des zerebralen Kortex (Maus), der Strukturdarstellung des Lungenparenchyms (Schwein) und des strukturellen Aufbaus von Knochentrabekeln (Schaf) in Abgrenzung zur Mikro-CT vorgestellt. Die vorliegende Übersichtsarbeit zeigt das Potenzial der Nano-CT als radiologische Methode in der biomedizinischen Grundlagenforschung und diskutiert die Nutzung hochauflösender, experimenteller CT Techniken unter Berücksichtigung alternativer Schnittbildverfahren. 


\section{Introduction}

$\nabla$

Regardless of the rapid technical development of multidetector CT in relation to high temporal resolution, this cross-sectional imaging method used in the clinical routine has a maximum spatial resolution of $240-600 \mu \mathrm{m}$. First in-vivo scanners, so-called high-resolution peripheral quantitative CT scanners (HR-pQCT), with a spatial resolution between 105 and $55 \mu \mathrm{m}$ at $10 \%$ MTF (XtremeCT (II), SCANCO Medical, Brüttisellen, Switzerland) are already in use for the visualization of the fine structure of peripheral bone [1]. Structures exceeding this achievable resolution currently cannot be visualized in detail in clinical cross-sectional CT imaging. Therefore, histology/pathology is still considered the gold standard for the visualization and quantification of structures with a size of less than $200 \mu \mathrm{m}$. This means that in addition to the time and personnel-intensive specimen preparation there are disadvantages regarding inexact or incomplete three-dimensional structure visualization and the destructive character of sample analysis. Micro-CT has become established as a complement to histological examination as a non-destructive three-dimensional imaging technique on the microscopic level. The following should be noted: There is no standard definition of the term "micro-CT". Kalender et al. [2] suggest a spatial resolution of at least $100 \mu \mathrm{m}$. CT scanners with higher spatial resolution can be referred to as micro-CT scanners regardless of the specific model. As a result of the acquisition of high-resolution image datasets with isotropic voxel geometry and an average spatial resolution of $5-50 \mu \mathrm{m}$, this technique provides a three-dimensional view of different organ systems and can provide information in addition to histomorphometry. This method was used primarily as part of osteological issues regarding the morphometry of compact and spongy bone [3]. The development of new contrast enhancement techniques could significantly expand the spectrum of possible applications. By using intravascular polymerizing contrast agents, knowledge of basic vascular research can be attained and pathological vascular changes can be visualized and quantified with high accuracy [4]. In-vivo micro-CT became established as a functional diagnostic method for pulmonary and cardiac performance parameters in small animal models $[5,6]$ and allows examination series with a longitudinal study design. Nonetheless, micro-CT, both in-vivo and ex-vivo, is limited by spatial resolution. These limits become evident in particular in fine structure imaging, e.g. in the visualization of terminal vessels, components of atherosclerotic plaques and cellular lacunae in calcified hard tissue. Building on the further development of micro-CT technology, nano-CT systems that differ partially with respect to design and performance (spatial resolution, tube voltage, and tube power) could be introduced (Xradia 800 Ultra: Zeiss, Oberkochen, Germany; nano-CT 2011, nano-CT 2211: Bruker MicroCT, Kontich, Belgium; Nanotom S, Nanotom M: GE/Phoenix X-ray, Wunstorf, Germany). By reducing the focal spot size and using suitable detectors and examination protocols (geometric enlargement, angular scanning, noise reduction by repetitive image acquisition), these systems allow imaging in the submicrometer range which is why the term nano-CT was introduced to differentiate from micro-CT. The goal of this overview is to describe technical device fundamentals, sample mounting, and application examples of nano-CT by way of example.

\section{Basics of nano-CT technology}

\section{7}

\section{X-ray generation}

High-resolution computed tomography in the submicrometer range has primarily been a domain of synchrotronbased micro-computed tomography (synMCT) [7, 8]. Based on the high achievable photon density and the monoenergetic X-ray radiation, the image quality is to be considered the gold standard due to the high signal-to-noise ratio and the lack of beam-hardening artifacts. However, synMCT does not represent an independent device class. Rather it is a method that is practiced at synchrotron facilities, i.e. major research institutions, such as the German Electron Synchrotron (DESY), the Conseil Européen pour la Recherche Nucléaire (CERN), and the National Synchrotron Light Source (NSLS). In contrast, the nano-computed tomography system (nano-CT 2011) presented in the following can be referred to as a prototype of an independent high-resolution device class. It is characterized by compact individual machines (standalone technology).

In the presented system the X-ray tube does not have a constant vacuum which is why a vacuum $\left(6-9 \times 10^{-4} \mathrm{~Pa}\right)$ is created via a suction pump and must be kept consistently stable ("open pumped type X-ray source"). The coil of the filament cathode is made of lanthanum hexaboride $\left(\mathrm{LaB}_{6}\right)$. Two horizontally and vertically connected condenser/objective electron lenses focus the emitted electrons. The anode is comprised of a tungsten-vaporized beryllium glass plate on which X-ray radiation is generated at acceleration voltages of $20-80 \mathrm{kVp}$ and a tube current of $100-200 \mu \mathrm{A}$. Due to the lack of collimation, no useful beam with a detectoradapted form is generated in the presented system and the aperture angle of the radiation is approx. $170^{\circ}$ (manufacturer information: Mars-Tohken, Tokyo, Japan). The horizontal and vertical divergence of the radiation results in coverage of the detector whose exposure geometry is analogous to that of flat detector/cone beam CT. Depending on the targeted spatial resolution/magnification, focal spot sizes between $<400$ and $600 \mathrm{~nm}$ can be selected, applications with a voxel side length in the submicrometer range typically being realized with the smallest possible focus $(<400 \mathrm{~nm})$. Compared to most microfocus X-ray tubes (classic reflection/direct beam tubes) used in micro-CT, these are transmission tubes, i.e., the X-ray window and focal spot coincide ( $\triangle$ Fig. 1 ). Since the object to be examined can be positioned closer to the focal spot and the diameter of the nano-focused focal spot is significantly smaller than in conventional micro-CT, a maximum spatial resolution in the submicrometer range is achieved. However, the thermal energy resulting from X-ray generation cannot be actively deflected from the focal spot which is why the tube current is significantly lower than in micro-CT and also differs between the selectable focal spot sizes. As a result, the photon density is lower than in micro-CT. Greater image noise occurs particularly at the smallest focus. In addition, greater enhancement of the detector signal is necessary due to the lower space-charge effect of the X-ray tube at a lower voltage, which also causes increased noise. Based on Kalender et al. [9], $\bullet$ Table 1 shows major differences between in-vivo and ex-vivo micro-CT and nano-CT. 


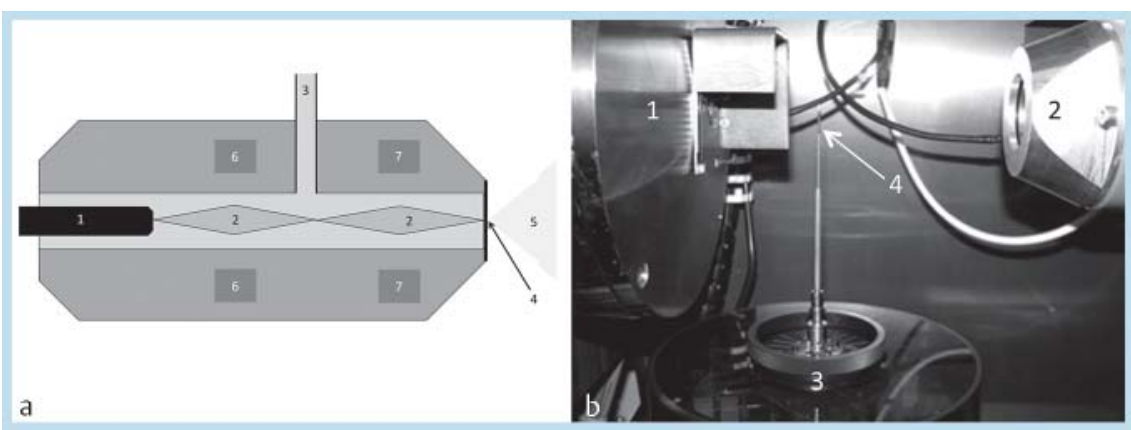

Fig. 1 a Schematic, not true to scale design of the transmission tube: Electron beam (2) emitted from the lanthanum hexaboride cathode (1). The vacuum in the tube is maintained by the connection (3) of a downstream vacuum pump (not shown). The X-ray window and the X-ray focus are comprised of a beryllium glass plate with a vapor-deposited tungsten layer (4). The generated radiation (5) is not collimated. The emitted radiation therefore does not have a cone geometry that is adapted to the detector but rather an opening angle of approx. $170^{\circ}$ (manufacturer specifica- tions). The nano focusing of the $\mathrm{X}$-ray beam is made possible by controllable pairs of electromagnetic condenser lenses (6) and electromagnetic objective lenses (7). b Rotating platform (3) with mounted sample holder (4) positioned between X-ray tube (partially shown) with X-ray window (1) and camera unit (2). By shifting along the Z-axis the desired geometric magnification is achieved, while the sample can be centered in the field of view by lateral and vertical movements.

\begin{tabular}{|c|c|c|c|}
\hline & in-vivo micro-CT & in-vitro micro-CT & nano-CT \\
\hline tube design & reflection tube & $\begin{array}{l}\text { reflection tube/ } \\
\text { transmission tube }\end{array}$ & transmission tube \\
\hline focus size & $50-200 \mu \mathrm{m}$ & $1-50 \mu \mathrm{m}$ & $<400 \mathrm{~nm}$ \\
\hline tube voltage & $20-90 \mathrm{kVp}$ & $20-130 k V p^{1}$ & $8-190 k V p$ \\
\hline tube power & $10-300 W$ & $1-50 W^{2}$ & $8-25 W$ \\
\hline spatial resolution ( $10 \%$ MTF) & $50-200 \mu \mathrm{m}$ & $5-100 \mu \mathrm{m}$ & $50-600 \mathrm{~nm}$ \\
\hline scan times & $0.1-30 \mathrm{~min}$ & $10-300 \mathrm{~min}$ & $60-180 \mathrm{~min}$ \\
\hline detector & CCD/flat panel detector & CCD/flat panel detector & CCD/flat panel detector \\
\hline
\end{tabular}

Table 1 Technical characteristics of in-vivo and in-vitro micro$\mathrm{CT}$ and presented nano- $\mathrm{CT}$ based on Kalender et al. [9].

The tube voltage ${ }^{1}$ or power ${ }^{2}$ used in biomedical in-vitro diagnosis is significantly lower than the power used for industrial measurement purposes that can exceed $1 \mathrm{MVp}$ (linear accelerator, not microfocused) or $500 \mathrm{~W}$.

\section{Image creation}

The sample fixed to an air-suspended rotating platform via a sample holder can be adjusted in all three spatial directions with a precision of up to $100 \mathrm{~nm}$. Depending on the selected magnification, the rotation movement can be freely defined in angle increments up to $0.1^{\circ}$ and scanning is performed over $180^{\circ}$ plus horizontal fan angle $\left(=185.15^{\circ}\right)$. Projection images are acquired using the "step and shoot" technique. This procedure is necessary to allow noise reduction by additional frame averaging at exposure times of $2 \mathrm{f} / \mathrm{s}$. At a fixed focus-detector distance (FDD) of $152 \mathrm{~mm}$, the rotating platform is mounted on a base that can move in the Z-direction between the focal spot/X-ray window and detector ( $\bullet$ Fig. 1 ) which determines the focus-object distance (FOD). The resulting geometric magnification (FDD/FOD) is between $\mathrm{M}_{\max }=52.1$ at $2.92 \mathrm{~mm}$ FOD and $\mathrm{M}_{\min }=1.1$ at $138.4 \mathrm{~mm}$ FOD. The image generation system is comprised of a terbium-doped gadolinium oxysulfide scintillator layer $\left(\mathrm{GD}_{2} \mathrm{O}_{2} \mathrm{~S}: \mathrm{Tb}\right)$ with a thickness of $15 \mu \mathrm{m}$, an image intensifier (Hamamatsu Photonics, Hamamatsu, Japan), and a CCD camera (ICX 285, Sony Corporation, Tokyo, Japan) with a matrix of $1280 \times 1024$ pixels at a pixel size of $10.5 \mu \mathrm{m}$. The subsequent cross-sectional image reconstruction (NRecon, Bruker microCT, Kontich, Belgium) is based on a reconstruction algorithm according to Feldkamp et al. [10]. Even if the determination of Hounsfield scaling according to the manufacturer software is possible, the presented nano-CT system lacks suitable phantoms for calibration. Moreover, there are significant differences between consecutive scans due to the filament properties, i. e., electron emission that fluctuates over time. Therefore, calibration based on one scan does not allow reliable transferability to subsequent examinations. The cross-sectional image datasets are therefore reconstructed and stored without calibration from a preselected gray value range of the projection dataset with gray value scaling of 8 bits as a bitmap (BMP) or 16 bits as tagged image file format (TIFF).

\section{Sample mounting}

To minimize truncation artifacts, the projection image of the sample should only minimally laterally exceed the detector. For optimal utilization of the detector, this means that the axis of rotation of the object moves near the vertical center of the detector. Axis deviations can be corrected by incremental fine adjustment of the rotating platform during examination preparation which allows continuous centering of the sample in front of the detector. Since detector size, object centering, and the required spatial resolution limit the maximum diameter of the sample, imaging in the submicrometer range requires preparation of samples in the order of magnitude of $\leq 1 \mathrm{~mm}$ diameter. As technical tools, a binocular reflected-light microscope or magnifying glasses, preparation instruments, a cutting mat, cylindrical punches, and wax paper (to protect against drying out and for securing on the sample holder) are virtual necessities for the preparation of samples. 


\section{Application spectrum of nano-CT}

$\nabla$

\section{Examination material}

The image material presented in the following comes from approved animal experiments (aorta experiment number: GI 20/9 no.106/2011; brain experiment number: GI 20/9 no.107/2011) or killings for non-scientific purposes (porcine lung, ovine bone). Examinations for comparative purposes were performed on a multidetector CT scanner (SOMATOM Definition, Siemens, Erlangen) or two micro-CT scanners (type 1072 and 1173) and a nano-CT scanner (type 2011, Bruker microCT, Kontich, Belgium). The technical differences among high-resolution systems are summarized in $\bullet$ Table 2.

\section{Imaging of atherosclerotic plaques in a small animal model}

The animal model of the $\mathrm{ApoE}_{(-/)} / \mathrm{LDLR}_{(-/)}$double knockout mouse (AL mouse) has become established in the last decade as an essential small animal model in atherosclerosis research [11]. Already 15 weeks after birth, atherosclerotic plaques can be seen in the descending aorta and in the aortic arch [12]. The first cup-shaped calcified plaques in the aortic arch and in the supraaortic vessels are seen after 20 weeks. Features of vulnerable lesions (plaque hemorrhage and increased vasa vasorum (VV) neovascularization) can be primarily seen in the descending aorta after 80 weeks. A quantitative evaluation of VV neovascularization could be repeatedly performed in micro-CT $[13,14]$. Plaque hemorrhages were primarily detected in synMCT [15]. In previous studies regarding plaque differentiation of atherosclerotic vascular wall lesions, quantitative statements regarding plaque composition differentiating fibrous, calcified, and lipid-rich plaques could be made in micro-CT studies [16]. The distribution, size, and type of atherosclerotic plaque can also be detected and histologically verified via nano-CT equivalent to micro-CT. Calcifying, fibrotic, and hemorrhagic plaques can be reliably differentiated on the basis of the vasa vasorum density, the X-ray opacity, and the object size [17]. Based on the improved spatial resolution in nano-CT, post-hemorrhagic iron accumulations can be identified on the basis of their fine-granular morphology ( $\bullet$ Fig. 2 ). In contrast to calcified plaques, grouped, point-shaped hyperdensities are seen in soft plaques located apart from the vasa vasorum neovascularization. While in-situ micro-CT ensures reliable detection of calcified plaques, there are clear deficits in the visualization of the internal structure. Imaging in the submicrometer range via nano-CT makes it possible to detect non-calcified recesses of single cells within the plaques ( 0 Fig. 3 ) whose histopathological correlate corresponds to matrixproducing chondrocyte-like cells.

\section{Imaging of cerebral vascularization}

Vascularization of the cerebral cortex shows hierarchical structuring in micro-CT. Radial blood conduits (cortical veins) drain into superficial veins (superficial cerebral veins) that are then directed into the sinus via bridging veins. Depending on the spatial resolution, horizontally situated fine parenchymal vessels in the sense of capillaries (nano-CT, $\odot$ Fig. 4) can be detected in addition to radially running larger parenchymal blood conduits (micro-CT). In addition to the improved detectability of the details of the smallest structures, the edge sharpness of the visualized objects increases regardless of the individual size. This results in more precise binarization which is mandatory for morphometry techniques for the automatic calculation of vessel volume, diameter, separation, etc. [18].

\begin{tabular}{|llll|}
\hline & type $\mathbf{1 0 7 2}$ & type 1173 & nano-CT 2011 \\
\hline tube type & reflection tube & reflection tube & transmission tube \\
\hline focus size & $8 \mu \mathrm{m}$ & $<5 \mu \mathrm{m}$ & $<400-600 \mathrm{~nm}$ \\
\hline tube voltage & $80 \mathrm{kVp}$ & $40-130 \mathrm{kVp}$ & $20-80 \mathrm{kVp}$ \\
\hline detector & $\mathrm{CCD}$ & flat panel detector & $\mathrm{CCD}$ \\
\hline spatial resolution $(10 \% \mathrm{MTF})$ & $15 \mu \mathrm{m}$ & $7 \mu \mathrm{m}$ & $400 \mathrm{~nm}$ \\
\hline
\end{tabular}

Table 2 Technical characteristics of the micro-/nano-CT devices used in Fig. $2-7$. Overview of tube and detector properties and the maximum achievable spatial resolution.

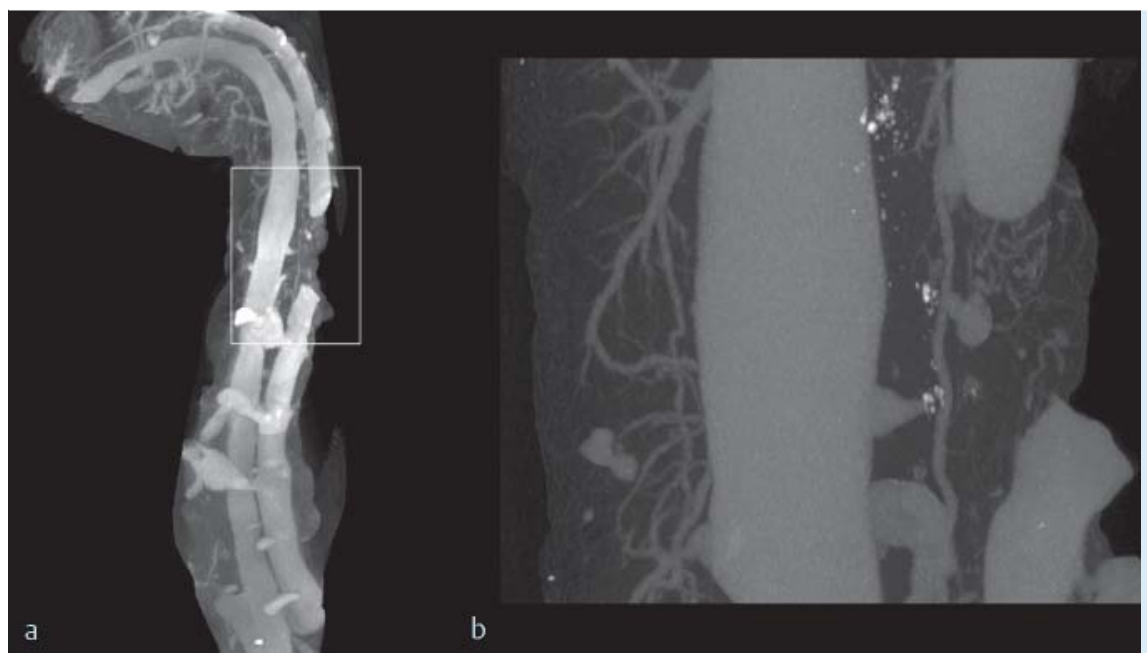

Fig. 2 Maximum intensity projection (MIP) of the descending aorta of a 78-week-old AL mouse: a Micro-CT (type 1072), imaging at $13.6 \mu \mathrm{m}$ isotropic voxel side length, $\mathbf{b}$ nano-CT: Enlarged image at $2.5 \mu \mathrm{m}$ isotropic voxel side length. Improving the spatial resolution makes the vasa-vasorum neovascularization clearer. In addition, the smallest points of opacity can be detected (histologically verified as iron content) as proof of plaque stage $\mathrm{V}$ or VIII, i. e., fibromuscular or fibrotic [30] after a hemorrhage (stage Vlb), in terms of an advanced lesion. 

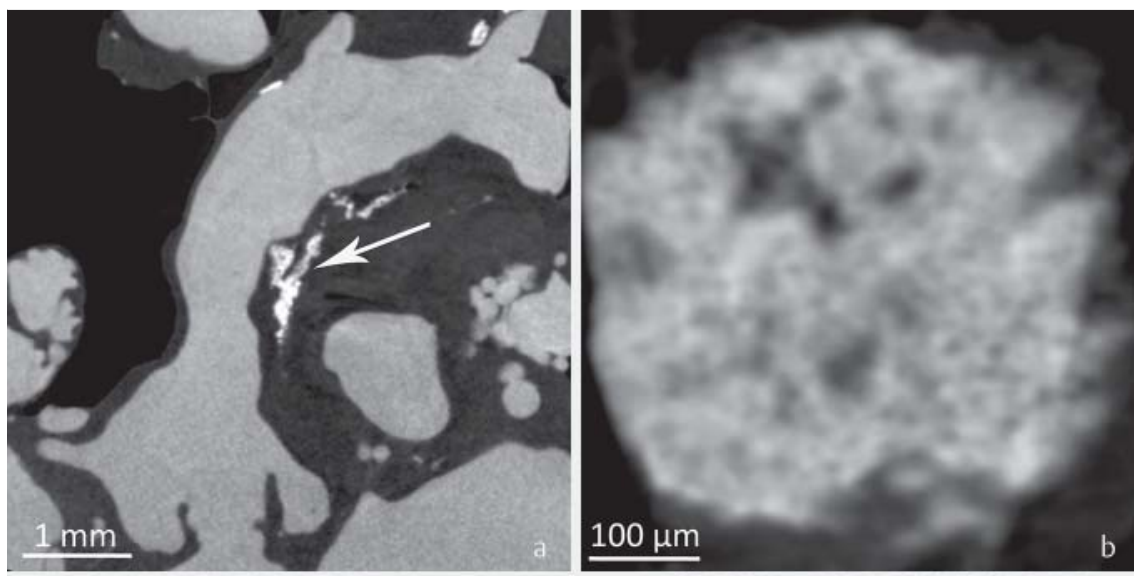

Fig. 3 Calcified atherosclerosis of the ascending aorta of a 72-week-old AL mouse: a Micro-CT of the heart-aorta preparation (type 1173) at an isotropic voxel side length of $7.6 \mu \mathrm{m}$ with calcified plaque (arrow) in the ascending aorta. Included are the branch of the right coronary artery and the aortic valve. $\mathbf{b}$ After removal of a calcified plaque, the examination is performed again with the same system at a maximum resolution of $5.6 \mu \mathrm{m}$ isotropic voxel side length. The internal structure of non-calcified areas can only be insufficiently differentiated. Noncalcified cell recesses cannot be detected. c Nano$\mathrm{CT}$ of the same calcified plaque is examined at $750 \mathrm{~nm}$ isotropic voxel side length. By detecting non-calcified cell recesses, chondrocyte-like cells can be identified and can then be verified by histol-
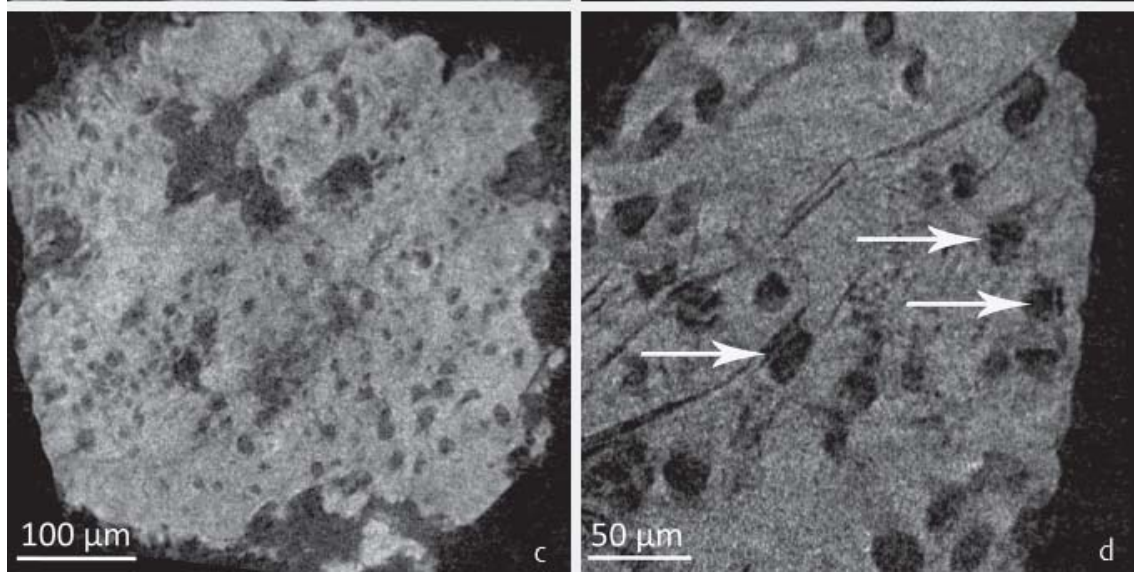
ogy [14]. $\mathbf{d}$ By increasing the spatial resolution (300 $\mathrm{nm}$ isotropic voxel side length), the cell recesses (arrows) and non-calcified linear plaque components can be shown more clearly.

\section{Imaging of fixed porcine lungs}

Micro-CT and synMCT are well established techniques in lung imaging and structure analysis [19]. By using monoenergetic, high-intensity synchrotron radiation, the fine structure of the lung can be visualized with minimal artifacts and high image quality, thus allowing anatomical fine structure analyses, e.g. of acinar lung structures $[20,21]$. While micro-CT requires the use of metal-containing contrast agents (e.g. osmium tetroxide) for contrast enhancement of parenchymal structures, high-resolution nano-CT allows visualization of alveolar structures without additional contrast enhancement and suppresses interfering partial volume effects. This facilitates binarization that is then the foundation of software-based structure analysis or morphometry techniques. However, this advantage is not applicable as a result of shifting of the spatial resolution into the application range of micro-CT. Use of the nano-CT technique does not show any advantages here ( $\bullet$ Fig. $\mathbf{5}$ ).

\section{Imaging of trabecular cell structures}

As opposed to clinically established multidetector CT, micro-CT is highly suitable for the visualization and characterization of trabecular networks and represents an established standard in preclinical osteoporosis research [22]. By also examining calcium hydroxyapatite phantoms, the bone mineral density (BMD) or the tissue mineral density (TMD) can be determined analogously to clinical QCT. For cellular regulation of bone metabolism, osteoblasts and osteoclasts as well as the population of osteocytes are increasingly becoming the focus of scientific interest [23-25]. In the case of an average osteocyte lacunae volume of approx.
$400 \mu \mathrm{m}^{3}[26,27]$ and under the assumption of a spherical shape, i. e., a diameter of approx. $9 \mu \mathrm{m}$, it quickly becomes clear that conventional micro-CT systems (see $\bullet$ Table 1 ) reach their limit here. The increase of the spatial resolution as a result of using nano-CT allows detection and morphometry of individual osteocyte lacunae and description of the spatial distribution in mineralized bone ( $\bullet$ Fig. 6,7 ).

\section{Conclusion}

$\nabla$

Imaging methods including the classic imaging techniques of radiology (CT/MRI) are an inherent component of basic biomedical research. The improved detection of details demonstrated for nano-CT makes it possible to address issues that could not be or could only be insufficiently examined under the previous device-specific resolution limits in computed tomography. The visualization of soft cellular lacunae in calcified tissues $[17,28]$ provides information that characterizes the interaction between cells and an extracellular matrix in the three-dimensional space. In addition, it shows the necessity for the development and researching of new contrast agents that allow differentiation of cells and the extracellular matrix in soft tissue, i.e., in the low contrast range [29]. The imaging of plaque hemorrhages indicating complicated or unstable atherosclerotic plaques was previously not possible via tube-based polychromatic micro-CT and was reserved for synchrotron-based monochromatic micro-CT. Thanks to the improved resolution in nano-CT, the visualization of histopathologically verifiable plaque hemorrhages in the form of ultra-fine iron particles 

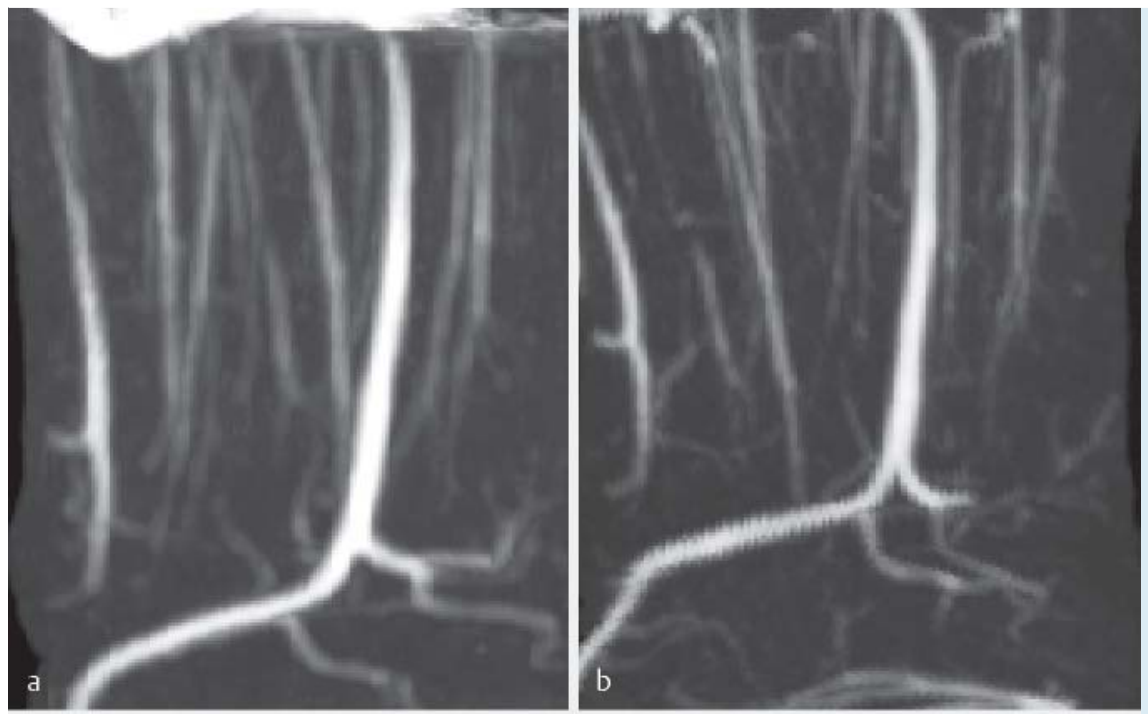

Fig. 4 MIP of vascularization of the murine cerebral cortex at $11.5 \mu \mathrm{m}$ isotropic voxel side length ac or $2 \mu \mathrm{m}$ isotropic voxel side length $\mathbf{d}$ : a Type 1072 , b type 1173, c type 2011, d enlargement scan, the same device as in image $\mathbf{c}$. At the same spatial resolution a-c there are small differences in the ability to detect details to the disadvantage of nano-CT c, a.e. due to the low tube power. The increase in spatial resolution $\mathbf{d}$ results in significantly improved detailed resolution with verification of the smallest horizontal vessels connected to the radially oriented larger-caliber vessels.
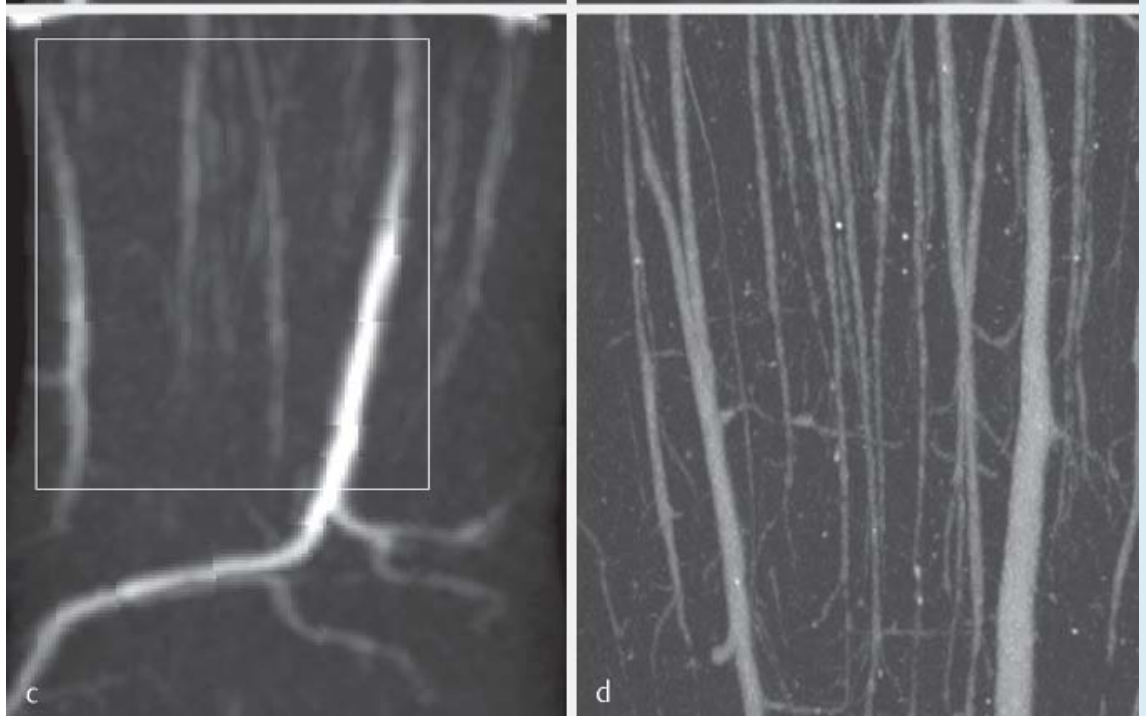

is now possible and provides information for the imaging of vulnerable plaques. The following is seen: Clinically relevant issues, e.g. precise plaque characterization [30], can already be addressed today from a technical standpoint, i.e., via nano-CT. Nano-CT is part of a spectrum of high-resolution non-destructive examination methods. In particular, optical and acoustic techniques such as confocal laser scanning microscopy (LSCM), optical coherence tomography (OCT), or ultrasound (ultrasound biomicroscopy - UBM) also allow cross-sectional imaging-based three-dimensional visualization and characterization of microscopic structures. As a result of rasterized scanning, LSCM acquires a three-dimensional dataset that shows the distribution of biomolecules, e.g. via temporary fluorescent marking. Thanks to examination times is the (sub-) second range, dynamic processes in living cells can be visualized, but other basic requirements than for nano-CT are valid for a sufficient examination. This includes a lower maximum thickness (approx. $100 \mu \mathrm{m}$ ) and lowest possible light scattering of the examination object. The surface topography, but not the internal structure, of opaque and light-scattering samples can be evaluated [31]. Since the best possible lateral spatial resolution and the Z-resolution (200 nm vs. $700 \mathrm{~nm}$ ) differ [32], voxel anisotropy occurs at maximum resolution.
In contrast to LSCM, the axial resolution of OCT is defined by the capacity, i.e., bandwidth and central wavelength of the light source, and today achieves resolution in the range of $500 \mathrm{~nm}$ and examination times in the sub-second range (swept source optical coherence tomography - SS-OCT) [33]. There are limitations for the examination of opaque, highly reflective structures, such as metallic stents [34, 35] that do not apply for micro-CT or nano-CT [29, 36]. As a real-time procedure, ultrasound biomicroscopy (UBM) achieves a spatial resolution of approx. $15 \mu \mathrm{m}$ (axial) or $30 \mu \mathrm{m}$ (lateral) [9, 37] at sound frequencies of up to approx. $100 \mathrm{MHz}$ with a tissue-dependent penetration depth of approx. $1-2 \mathrm{~mm}$ which corresponds to a significantly lower spatial resolution compared to nano-CT at a comparable FOV. For the spectrum of higher sound frequencies (scanning acoustic microscopy -SAM - up to $2 \mathrm{GHz}$ ), mapping of mechanical properties $[38,39]$ with spatial resolution of less than $100 \mathrm{~nm}$ can be achieved. Sample preparation is more complex. While UBM can be applied in vivo, slice creation prior to examination is recommended for SAM [40]. In addition, the use of suitable coupling media (degassed water) has a certain disadvantage since dry preserved samples can be damaged. 


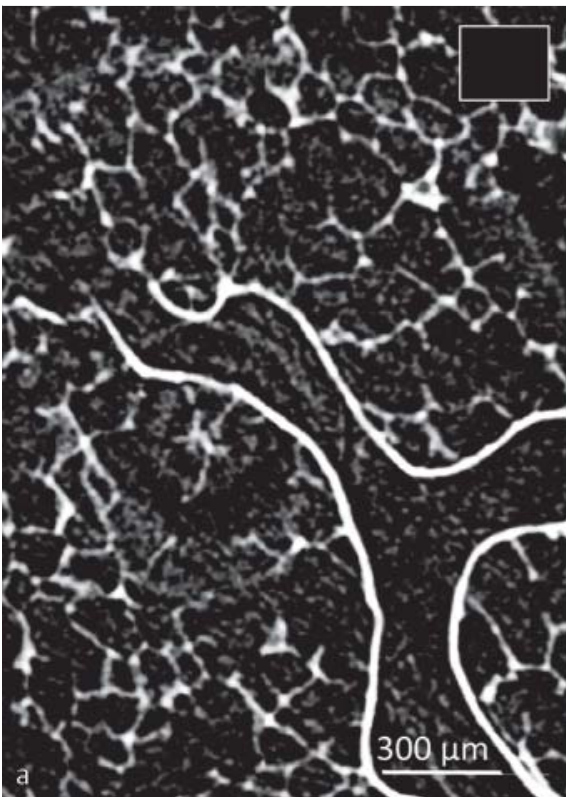

Fig. 5 In porcine lung parenchyma fixed in formalin vapor without additional contrast enhancement in micro-CT (type 1173) or in nano-CT at $5.6 \mu \mathrm{m}$ isotropic voxel side length $\mathbf{a}, \mathbf{b}$ or in nano-CT at $750 \mathrm{~nm}$ isotropic voxel side length $\mathbf{c}$. Regardless of device type, structures distal to the terminal bronchioles are imaged in a noisy and unclear manner at a spatial reso-

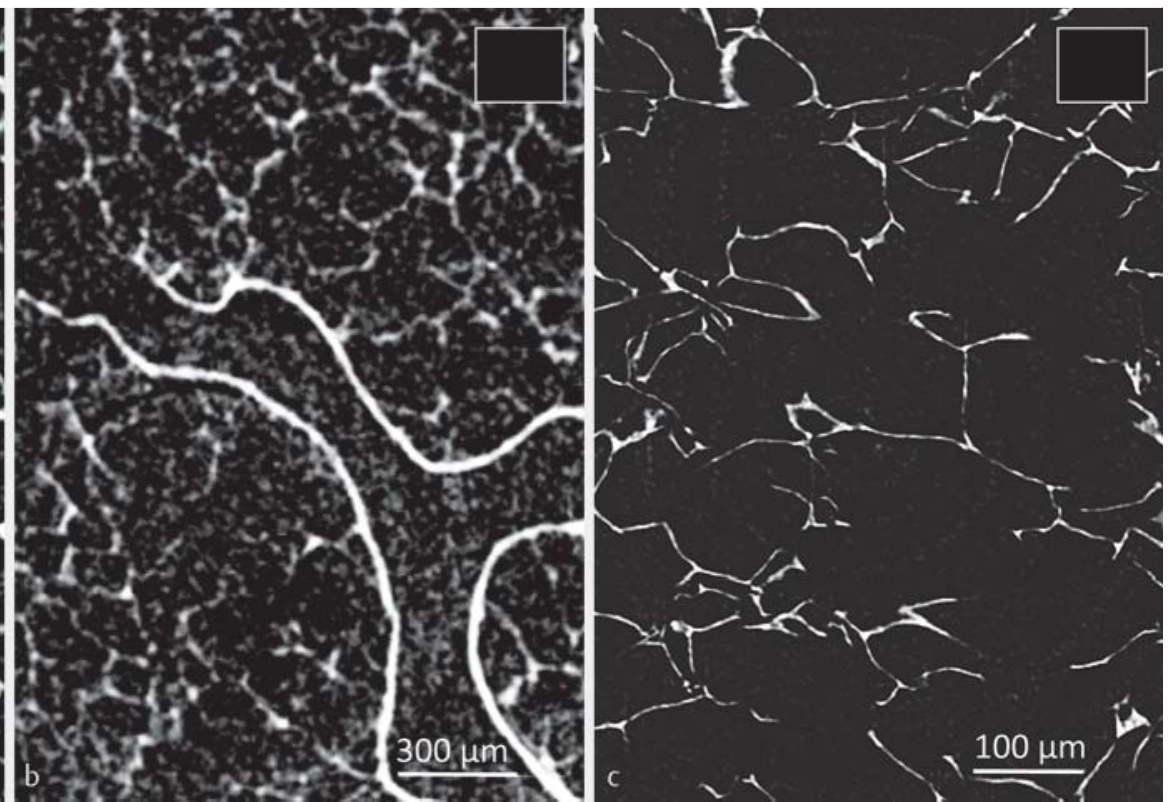

lution in the micrometer range $\mathbf{a}, \mathbf{b}$ due to the low absorption and the minimal thickness. As a result of increased spatial resolution in nano-CT, alveolar structures can be clearly defined even without prior contrast enhancement.
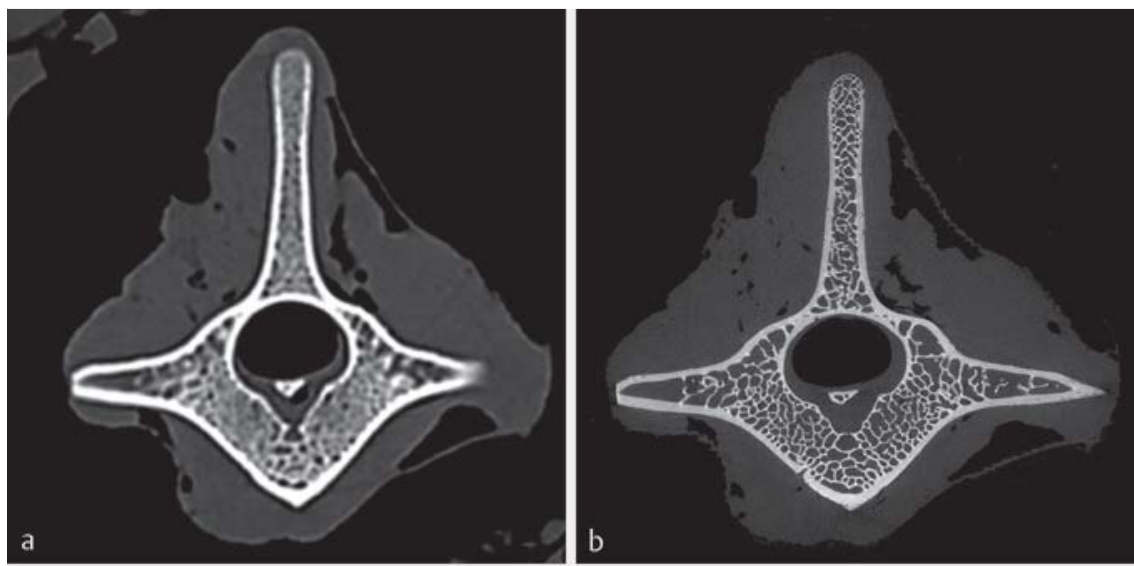

Fig. 6 After examination of an ovine spinal column preparation $\mathbf{a}$ in MDCT and $\mathbf{b}$ in micro-CT (type $1173,33 \mu \mathrm{m}$ istotropic voxel edge length), an individual trabecula was removed. The examination in micro-CT at $5.6 \mu \mathrm{m}$ isotropic voxel side length $\mathbf{c}$ was followed by the comparative examination in nano$\mathrm{CT}$ at $600 \mathrm{~nm}$ isotropic voxel side length $\mathbf{d}$. Despite increasing resolution in micro-CT of up to the device-specific resolution limit c, osteocyte lacunae cannot be delimited. Imaging in nano-CT on the sub-micrometer level allows a look at the trabecular microstructure and visualizes osteocyte lacunae and trabecular vascular canals.
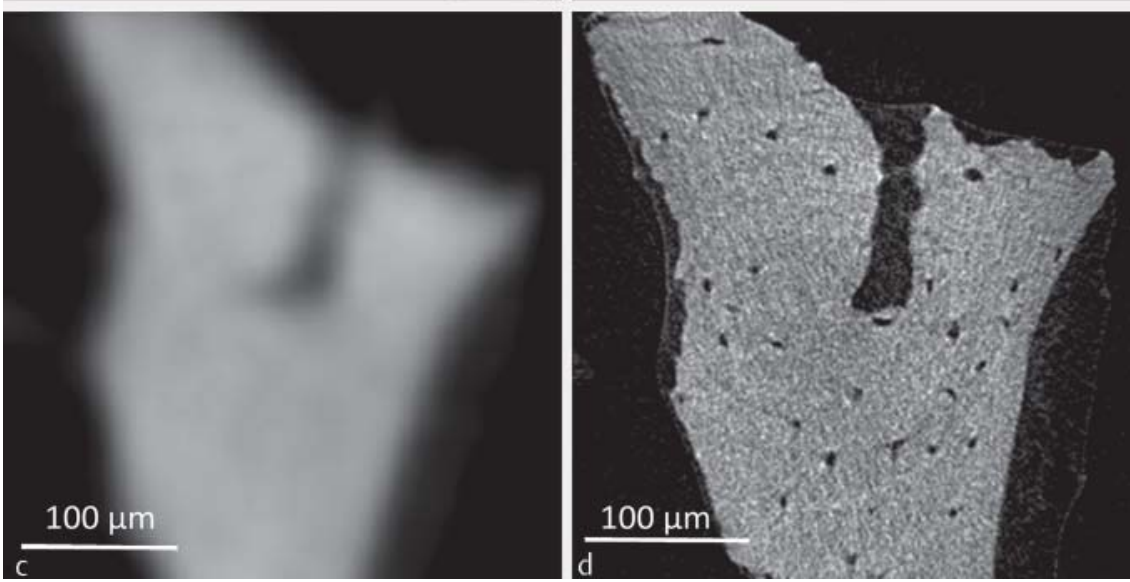

Typical limitations of the method must also be taken into consideration for nano-CT. The relatively long examination times compared to optical and acoustic methods allow a throughput of two to three examinations per day. Moreover, the size of samples must be small when a spatial resolution in the sub-micrometer range is targeted (see sample mounting). Solutions for this were developed in previous years: As a result of the use of powerful tubes and detectors 

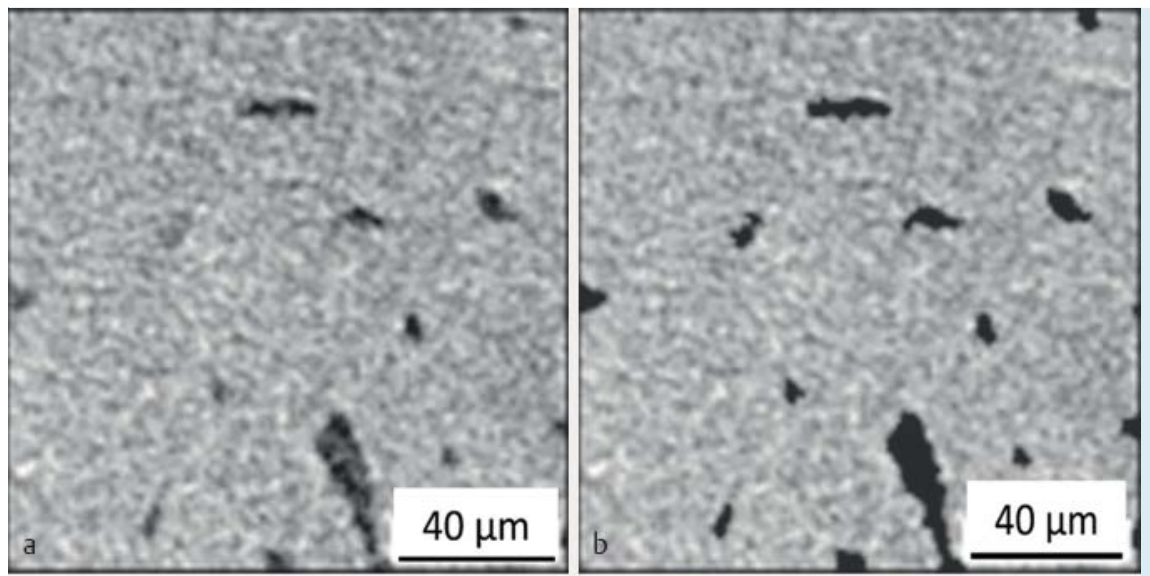

Fig. 7 Nano-CT of the osseous microstructure: a Grayscale image of a $150 \mathrm{~m} \times 150 \mathrm{~m}$ image section, istotropic voxel size of $600 \mathrm{~nm}$ in the case of an image matrix of $250 \times 250$ pixels. The osteocyte lacunae and an included intraosseous vessel have soft internal structures. $\mathbf{b}$ Image fusion of the mineralized bone substance (grayscale image) and the non-mineralized bone segments identified by adaptive threshold adjustment and displayed in black. c Inverted, binarized cross-sectional image with visualization of mineralized (black) or non-mineralized (white) bone segments. $\mathbf{d}$ Derived threedimensional visualized dataset with segmentation of the osteocyte lacunae (magenta) or of a vascular
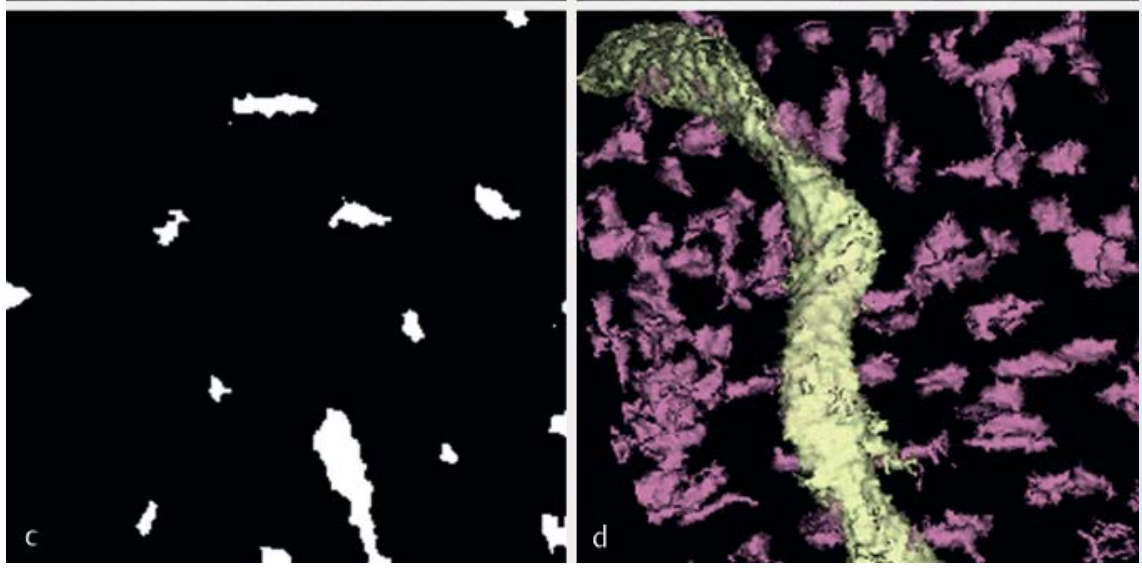
canal (yellow) in volume-rendering technique, VRT.

(flat panel detectors to $3072 \times 2400$ pixels: Nanotom M) and techniques for enlarging the field of view by lateral shifting of the detector (offset technique: SkyScan 2211), relatively large objects can be examined in nano-CT. Moreover, the improvement of existing reconstruction techniques makes it possible to suppress truncation artifacts which makes artifact-free examination of defined regions within an object (ROI scanning) possible [41-43]. The associated improvement for fine structure analysis of larger objects highlights the non-destructive examination character of the method since the preparation effort, i.e., manipulation of the samples, can be reduced in advance.

\section{Limitations}

In contrast to micro-CT, in-vivo use of nano-CT does not seem promising for now. The necessary suppression of motion artifacts from physiological movement patterns, e.g. from heartbeat, breathing, and intestinal movement, would require significantly shorter image acquisition times and higher tube power. Thus the technique is to be viewed also perspectively as an imaging method of ex-vivo analysis.

\section{Future possibilities}

The application of nano-CT in experimental ischemia diagnosis and therapy allows improved visualization of cerebral microcirculation [44] to the capillary level. Use for fine structure imaging of the osseous [28, 45], renal [46, 47], and dental [48] anatomy and pathophysiology as well as scaffold imaging [29] are examples for the potential of this radiological technique and expected uses in coming years.

\section{Acknowledgment}

$\nabla$

The authors would like to thank the German Research Society for support with respect to the special research area Transregio 79 (SFB/TRR 79 "materials for tissue regeneration within systemically altered bone").

\section{References}

1 Kocijan R, Finzel S, Englbrecht $M$ et al. Decreased quantity and quality of the periarticular and nonperiarticular bone in patients with rheumatoid arthritis: a cross-sectional HR-pQCT study. J Bone Miner Res 2014; 29: 1005 - 1014

2 Kalender WA. Special Applications. In: Kalender WA. Computed tomography fundamentals, system technology, image quality, applications 3. ed. Erlangen: Publicis Corp Publ; 2011: 272

3 Engelke K, Karolczak M, Lutz A et al. Micro-CT. Technology and application for assessing bone structure. Radiologe 1999; 39: 203 -212

4 Ortiz MC, Garcia-Sanz A, Bentley MD et al. Microcomputed tomography of kidneys following chronic bile duct ligation. Kidney Int 2000; 58: $1632-1640$

5 Badea C, Hedlund LW, Johnson GA. Micro-CT with respiratory and cardiac gating. Med Phys 2004; 31: 3324-3329

6 Lee CL, Min H, Befera $\mathrm{N}$ et al. Assessing cardiac injury in mice with dual energy-microCT, $4 \mathrm{D}$-microCT, and microSPECT imaging after partial heart irradiation. Int J Radiat Oncol Biol Phys 2014; 88: 686-693

7 Schneider P, Stauber M, Voide R et al. Ultrastructural properties in cortical bone vary greatly in two inbred strains of mice as assessed by synchrotron light based micro- and nano-CT. J Bone Miner Res 2007; 22: $1557-1570$

8 Pacureanu A, Langer M, Boller $E$ et al. Nanoscale imaging of the bone cell network with synchrotron X-ray tomography: optimization of acquisition setup. Med Phys 2012; 39: 2229-2238 
9 Kalender WA, Deak P, Engelke K et al. X-Ray and X-Ray CT. In: Kiessling F, Pichler BJ. Small Animal Imaging Basics and Practical Guide. Springer-Verlag Berlin Heidelberg; 2011: 129

10 Feldkamp LA, Davis LC, Kress JW. Practical Cone-Beam Algorithm. J Opt Soc Am A 1984; 1: 612-619

11 Jawien J, Nastalek P, Korbut R. Mouse models of experimental atherosclerosis. J Physiol Pharmacol 2004; 55: 503-517

12 Witting PK, Pettersson K, Ostlund-Lindqvist AM et al. Inhibition by a coantioxidant of aortic lipoprotein lipid peroxidation and atherosclerosis in apolipoprotein $\mathrm{E}$ and low density lipoprotein receptor gene double knockout mice. FASEB J 1999; 13: 667-675

13 Gossl M, Herrmann J, Tang $H$ et al. Prevention of vasa vasorum neovascularization attenuates early neointima formation in experimental hypercholesterolemia. Basic Res Cardiol 2009; 104: 695 - 706

14 Ritman EL, Lerman A. The dynamic vasa vasorum. Cardiovasc Res 2007; 75: 649-658

15 Langheinrich AC, Michniewicz A, Sedding DG et al. Quantitative X-ray imaging of intraplaque hemorrhage in aortas of apoE(-/-)/LDL(-/-) double knockout mice. Invest Radiol 2007; 42: 263-273

16 Langheinrich AC, Bohle RM, Greschus S et al. Atherosclerotic lesions at micro CT: feasibility for analysis of coronary artery wall in autopsy specimens. Radiology 2004; 231: 675-681

17 Kampschulte M, Brinkmann A, Stieger P et al. Quantitative CT imaging of the spatio-temporal distribution patterns of vasa vasorum in aortas of apoE-/-/LDL-/- double knockout mice. Atherosclerosis 2010; 212: $444-450$

18 Duvall CL, Taylor WR, Weiss D et al. Quantitative microcomputed tomography analysis of collateral vessel development after ischemic injury. Am J Physiol Heart Circ Physiol 2004; 287: H302 - H310

19 McDonough JE, Yuan R, Suzuki M et al. Small-airway obstruction and emphysema in chronic obstructive pulmonary disease. N Engl J Med 2011; 365: 1567-1575

20 Litzlbauer HD, Korbel K, Kline TL et al. Synchrotron-based micro-CT imaging of the human lung acinus. Anat Rec (Hoboken) 2010; 293: $1607-1614$

21 Watz H, Breithecker A, Rau WS et al. Micro-CT of the human lung: imaging of alveoli and virtual endoscopy of an alveolar duct in a normal lung and in a lung with centrilobular emphysema - initial observations. Radiology 2005; 236: $1053-1058$

22 Bouxsein ML, Boyd SK, Christiansen BA et al. Guidelines for assessment of bone microstructure in rodents using micro-computed tomography. J Bone Miner Res 2010; 25: 1468-1486

23 Carpentier VT, Wong J, Yeap Y et al. Increased proportion of hypermineralized osteocyte lacunae in osteoporotic and osteoarthritic human trabecular bone: implications for bone remodeling. Bone 2012; 50: $688-694$

24 Rochefort GY. The osteocyte as a therapeutic target in the treatment of osteoporosis. Ther Adv Musculoskelet Dis 2014; 6: 79-91

25 Schaffler MB, Kennedy OD. Osteocyte signaling in bone. Curr Osteoporos Rep 2012; 10: 118-125

26 Dong $P$, Haupert S, Hesse B et al. 3D osteocyte lacunar morphometric properties and distributions in human femoral cortical bone using synchrotron radiation micro-CT images. Bone 2014; 60: 172 - 185

27 Carter Y, Thomas CD, Clement JG et al. Variation in osteocyte lacunar morphology and density in the human femur - a synchrotron radiation micro-CT study. Bone 2013; 52: 126-132

28 van Hove RP, Nolte PA, Vatsa A et al. Osteocyte morphology in human tibiae of different bone pathologies with different bone mineral density - is there a role for mechanosensing? Bone 2009; 45: 321 - 329
29 Papantoniou I, Sonnaert M, Geris L et al. Three-dimensional characterization of tissue-engineered constructs by contrast-enhanced nanofocus computed tomography. Tissue Eng Part C Methods 2014; 20: 177 187

30 Stary HC. Natural history and histological classification of atherosclerotic lesions: an update. Arterioscler Thromb Vasc Biol 2000; 20: $1177-1178$

31 Helmchen F, Denk W. Deep tissue two-photon microscopy. Nat Methods 2005; 2 : $932-940$

32 Claxton NS, Fellers TJ, Davidson MW. Microscopy, Confocal. In: Encyclopedia of Medical Devices and Instrumentation. 2006

33 Podoleanu AG. Optical coherence tomography. J Microsc 2012; 247: 209-219

34 Bezerra HG, Costa MA, Guagliumi G et al. Intracoronary optical coherence tomography: a comprehensive review clinical and research applications. JACC Cardiovasc Interv 2009; 2: 1035-1046

35 Elahi S, Mancuso JJ, Milner TE et al. Sunflower artifact in OCT. JACC Cardiovasc Imaging 2011; 4: 1220 - 1221

36 Langheinrich AC, Zoerb C, Jajima J et al. Quantification of in-stent restenosis parameters in rabbits by Micro-CT. Fortschr Röntgenstr 2005; 177: $501-506$

37 Turnbull DH, Starkoski BG, Harasiewicz KA et al. A 40-100 MHz B-scan ultrasound backscatter microscope for skin imaging. Ultrasound Med Biol 1995; 21: 79-88

38 Petter-Puchner A, Gruber-Blum S, Walder $N$ et al. Ultrasound biomicroscopy (UBM) and scanning acoustic microscopy (SAM) for the assessment of hernia mesh integration: a comparison to standard histology in an experimental model. Hernia 2014; 18: 579-585

39 Raum K, Kempf K, Hein HJ et al. Preservation of microelastic properties of dentin and tooth enamel in vitro - a scanning acoustic microscopy study. Dent Mater 2007; 23: 1221 -1228

40 Maeva E, Severin F, Miyasaka C et al. Acoustic imaging of thick biological tissue. IEEE Trans Ultrason Ferroelectr Freq Control 2009; 56: $1352-1358$

41 Sen Sharma K, Gong H, Ghasemalizadeh $O$ et al. Interior micro-CT with an offset detector. Med Phys 2014; 41: 061915

42 Sen Sharma K, Holzner C, Vasilescu DM et al. Scout-view assisted interior micro-CT. Phys Med Biol 2013; 58: 4297-4314

43 Xia Y, Dennerlein F, Bauer S et al. Scaling calibration in region of interest reconstruction with the 1D and 2D ATRACT algorithm. Int J Comput Assist Radiol Surg 2014; 9: 345-356

44 Nedelmann $M$, Ritschel $N$, Doenges $S$ et al. Combined contrast-enhanced ultrasound and rt-PA treatment is safe and improves impaired microcirculation after reperfusion of middle cerebral artery occlusion. J Cereb Blood Flow Metab 2010; 30: 1712-1720

45 Vatsa A, Breuls RG, Semeins CM et al. Osteocyte morphology in fibula and calvaria - is there a role for mechanosensing? Bone 2008; 43 : $452-458$

46 Langheinrich AC, Kampschulte M, Scheiter F et al. Atherosclerosis, inflammation and lipoprotein glomerulopathy in kidneys of apoE-/-/ LDL-/- double knockout mice. BMC Nephrol 2010; 11: 18

47 Wagner R, Van Loo D, Hossler F et al. High-resolution imaging of kidney vascular corrosion casts with Nano-CT. Microsc Microanal 2011; 17: 215-219

48 Parkinson CR, Sasov A. High-resolution non-destructive 3D interrogation of dentin using X-ray nanotomography. Dent Mater 2008; 24: $773-777$ 